Monatsschr Kinderheilkd $2021 \cdot$ 169:900-901 https://doi.org/10.1007/s00112-021-01299-2 Angenommen: 2. August 2021

(c) Springer Medizin Verlag $\mathrm{GmbH}$, ein Teil von Springer Nature 2021

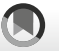

\title{
Diabetes mellitus im Kindes- und Jugendalter
}

\section{Gesine Hansen}

Klinik für Pädiatrische Pneumologie, Allergologie und Neonatologie, Zentrum Kinderheilkunde und Jugendmedizin, Hannover, Deutschland
Der Diabetes mellitus Typ 1 stellt die häufigste Stoffwechselerkrankung im Kindesund Jugendalter dar. Die Neuerkrankungsrate steigt um 3-4\% pro Jahr und beträgt aktuell ca. 33/100.000 Kinder im Alter zwischen 0 und 15 Jahren. Die Ursachen für diesen kontinuierlichen Anstieg der Inzidenz sind nicht bekannt. Im Gegensatz zu den Erwartungen hat die Inzidenz des Typ2-Diabetes bei Heranwachsenden in den letzten 10 Jahren nicht zugenommen und beträgt relativ konstant ca. 200 Neuerkrankungen/Jahr bei den unter 20-Jährigen. Bei dem Typ-1-Diabetes mellitus sind dagegen ca. 3000 Kinder und Jugendliche/ Jahr von einer Neuerkrankung betroffen. Die Diagnose des Typ-1-Diabetes stützt sich auf die klinische Symptomatik wie PoIydipsie, Polyurie und Gewichtsabnahme sowie die Ergebnisse der Blutzuckermessungen. Die klinischen Zeichen können aber auch sehr unspezifisch sein, wie z.B. ein reduzierter Allgemeinzustand, Bauchschmerzen, Erbrechen oder eine erschwerte Atmung. Eine spezialisierte pädiatrischdiabetologische Betreuung durch ein multiprofessionelles Team führt nachweislich zu einer besseren Kontrolle des Diabetes und einer Reduktion der Hospitalisierungsrate und sollte nach Möglichkeit immer angestrebt werden.

\section{॥) Fortschritte in Prävention und Therapie: spannende Themen in der pädiatrischen Diabetologie}

Der wesentliche Fortschritt in der Behandlung von Menschen mit einem Typ-1-Diabetes besteht neben neuen Insulinen in der stetigen Weiterentwicklung der technischen Möglichkeiten der Insulinapplikation. Insulinpumpen und Glukosesensoren werden von Kindern und Jugendlichen deutlich häufiger angewendet als von Erwachsenen; bei Kleinkindern beträgt die Nutzungsrate von Insulinpumpen bereits über $95 \%$. In dem Beitrag „Individualisierung der Diabetestherapie durch Automatisierung der Insulingabe" von Biester et al. werden die Möglichkeiten einer Individualisierung und Optimierung der Behandlung des Typ-1-Diabetes durch eine verbesserte, teils kombinierte und interaktive Nutzung von verschiedenen Technologien zur Insulingabe vorgestellt. Darüber hinaus werden Zukunftsperspektiven einer vollautomatischen Insulingabe aufgezeigt, die eine deutliche Erleichterung für die Patienten und eine weitere Optimierung der Diabetesbehandlung ermöglichen könnten.

Der Diabetes mellitus geht relativ häufig mit begleitenden Erkrankungen einher. In ihrem Beitrag „Komorbiditäten bei Typ1-Diabetes im Kindes- und Jugendalter" fasst Elke Fröhlich-Reiterer die physischen Komorbiditäten des Typ-1-Diabetes wie andere Autoimmunerkrankungen, eine verzögerte Pubertätsentwicklung, ein eingeschränktes Längenwachstum oder kardiovaskuläre Komplikationen zusammen, auf die bei der Betreuung von Patienten mit Diabetes mellitus besonders zu achten ist.

Mehr als $90 \%$ der Kinder und Jugendlichen mit einem Diabetes mellitus erkranken an dem autoimmun vermittelten Diabetes Typ 1; andere Diabetesformen sind vergleichsweise selten. Für den Therapieerfolg und die Prognose ist es von großer Wichtigkeit, diese anderen Diabetesformen frühzeitig zu erkennen und gezielt zu behandeln. In ihrem Beitrag "Nicht immer Typ 1 - Seltene Diabetesformen bei Kindern" gibt Katharina Warncke einen Überblick über diese seltenen Diabetes- 
formen und die damit verbundenen diagnostischen, therapeutischen und prognostischen Implikationen.

Der kontinuierliche deutliche Anstieg der Inzidenz des Diabetes mellitus Typ 1 bei Kindern und Jugendlichen spricht für einen starken Einfluss von Umweltfaktoren auf die Entstehung der Erkrankung. In ihrem Beitrag "Neue Wege zur Prävention des Typ-1-Diabetes bei Kindern - Chancen und Risiken" stellen Olga Kordonouri et al. neue Strategien zur populationsbasierten Früherkennung von Risikopersonen und innovative Studien zu neuen immunmodulatorischen Präventions- und Therapieansätzen vor. Ein Erfolg dieser großen internationalen Anstrengung mit der Beteiligung mehrerer deutscher Zentren könnte sehr weitreichende Implikationen für die Prävention dieser immer häufiger auftretenden Autoimmunerkrankung mit sich bringen.

Ich wünsche Ihnen viel Freude bei der Lektüre unseres Updates zum Diabetes mellitus und hoffe, dass Sie in den Beiträgen neue und spannende Informationen für Ihren klinischen Alltag entdecken werden.

Mit herzlichen Grüßen,

Ihre

Gesine Hansen

\section{Korrespondenzadresse}

\section{Prof. Dr. Gesine Hansen}

Klinik für Pädiatrische Pneumologie, Allergologie und Neonatologie, Zentrum Kinderheilkunde und Jugendmedizin Carl-Neuberg-Str. 1, 30625 Hannover, Deutschland

hansen.gesine@mh-hannover.de

Interessenkonflikt. G. Hansen gibt an, dass kein Interessenkonflikt besteht.

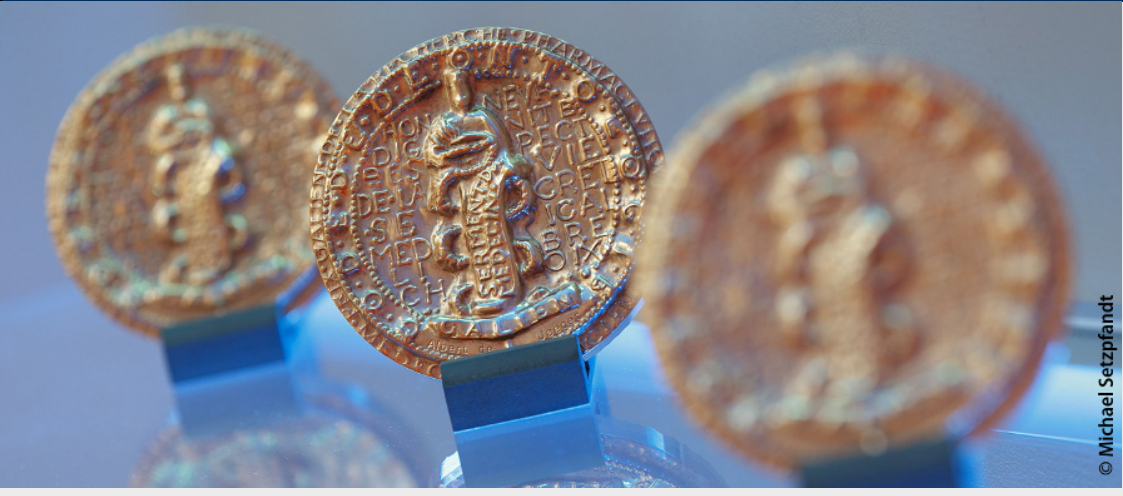

\section{Galenus-von-Pergamon-Preis 2021 - die Kandidaten Leqvio ${ }^{\circledR}$ - erste siRNA zur Hypercholesterinämie-Behandlung}

Inclisiran (Leqvio ${ }^{\circledR}$ ) von Novartis ist die bisher einzige small interfering RNA zur Hypercholesterinämie-Behandlung. Dieser Kandiat für den diesjährigen Galenusvon-Pergamon-Preis induziert eine starke und anhaltende Senkung von LDLCholesterin und muss in der Dauertherapie nur einmal alle sechs Monate subkutan appliziert werden.

LDL-Cholesterin (LDL-C) ist der wichtigste Treiber von atherosklerotischen kardiovaskulären Erkrankungen (atherosclerotic cardiovascular disease, ASCVD), den häufigsten Todesursachen in Deutschland. Je tiefer die LDL-C-Spiegel gesenkt werden, desto ausgeprägter ist der günstige Effekt. Im Versorgungsalltag erreichen jedoch die meisten Risikopatienten trotz optimierter oraler Therapie nicht die in den Leitlinien empfohlenen LDL-C-Zielwerte.

\section{Dauertherapie: Gabe alle sechs Monate}

Inclisiran (Leqvio ${ }^{\circledR}$ ) ist eine doppelsträngige kleine interferierende (small interfering) Ribonukleinsäure (siRNA). Sie hemmt die Bildung des Enzyms Proproteinkonvertase Subtilisin Kexin Typ 9 (PCSK9), das eine Schlüsselrolle im Cholesterinstoffwechsel spielt. Inclisiran wird nach einer initialen Dosis und einer weiteren Dosis nach drei Monaten dann in der Dauertherapie nur alle sechs Monate subkutan injiziert.

Inclisiran wird bei Erwachsenen mit primärer Hypercholesterinämie (heterozygot familiär und nicht familiär) oder gemischter Dyslipidämie zusätzlich zu diätetischer Therapie angewendet, in Kombination mit einem Statin oder einem Statin mit anderen lipidsenkenden Therapien bei Patienten, die mit der maximal tolerierten Statindosis die LDLC-Ziele nicht erreichen, oder allein oder in Kombination mit anderen lipidsenkenden
Therapien bei Patienten mit Statinintoleranz oder mit Kontraindikation für ein Statin.

Eine gepoolte Analyse der drei zulassungsrelevanten Studien ORION-9, -10 und -11 zeigte eine signifikante Senkung des LDLC-Spiegels: Mit der siRNA erreichten nach 17 Monaten $68 \%$ der Patienten einen LDLC-Wert von $70 \mathrm{mg} / \mathrm{dl}$ und $52 \%$ einen LDLC-Wert von $50 \mathrm{mg} / \mathrm{dl}$. Dabei war die LDL-CSenkung konsistent über alle Subgruppen hinweg. Darüber hinaus wurde die Reduktion von PCSK9, Apolipoprotein B, Nicht-HDL-C und Lipoprotein(a) dokumentiert.

Als einzige mit Inclisiran assoziierte unerwünschte Wirkungen traten leichte bis mäßige Reaktionen an der Injektionsstelle auf, die ohne Folgeschäden wieder abklangen.

\section{Infos zum Preis}

Mit dem Galenus-von-Pergamon-Preis Deutschland werden seit 1985 jedes Jahr herausragende Arzneimittel-Innovationen ausgezeichnet, die in Deutschland auf den Markt gebracht worden sind. Ziel des von der Springer Medizin Verlag GmbH gestifteten Preises ist es, die pharmakologische Forschung für innovative Arzneimittel zu fördern. Vergeben wird der Galenus-vonPergamon-Preis in den Kategorien "Primary Care", "Specialist Care" und "Orphan Drugs". Über die Zuerkennung entscheidet eine unabhängige Expertenjury. Die Verleihung findet am 21. Oktober $2021 \mathrm{im}$ Rahmen eines Festaktes in Berlin statt. (kw) 